\title{
Cognitive Spare Capacity as a Window on Hearing Aid Benefit
}

\author{
Mary Rudner and Thomas Lunner
}

\section{Linköping University Post Print}

\section{Tweet}

N.B.: When citing this work, cite the original article.

Original Publication:

Mary Rudner and Thomas Lunner, Cognitive Spare Capacity as a Window on Hearing Aid Benefit, 2013, Seminars in Hearing, (34), 298-307.

http://dx.doi.org/10.1055/s-0033-1356642

Copyright: Thieme Publishing / Georg Thieme Verlag http://www.thieme.com/

Postprint available at: Linköping University Electronic Press

http://urn.kb.se/resolve?urn=urn:nbn:se:liu:diva-105111 
Cognitive Spare Capacity

Cognitive Spare Capacity as a Window on Hearing Aid Benefit

$$
\text { Mary Rudner }{ }^{1} \text { and Thomas Lunner }{ }^{1,2,3}
$$

${ }^{1}$ Linnaeus Centre HEAD, Swedish Institute for Disability Research, Department of Behavioural Sciences and Learning, Linköping University, Sweden

${ }^{2}$ Department of Clinical and Experimental Medicine, Linköping University, Sweden

${ }^{3}$ Eriksholm Research Centre, Oticon A/S, Denmark

Contact information:

Mary Rudner

Department of Behavioural Sciences and Learning

Linköping University

SE-581 83 Linköping

Sweden

Tel. +4613282157

Fax. +4613282145

mary.rudner@liu.se 


\section{Cognitive Spare Capacity}

Abstract

It is well established that successful listening with advanced signal processing in digital hearing aids is associated with individual working memory capacity which is the cognitive ability to keep information in mind and process it. Different types of cognitive processing may be required in different situations. For example, when listening in noise it may be necessary to inhibit irrelevant information and update misheard information. There is evidence that simply hearing a spoken utterance consumes cognitive resources and may do so to different degrees for different individuals. In order to determine just how useful different kinds of signal processing are, it is important to determine to what extent they help individual hearing aid users cope with the kind of cognitive demands that may arise in everyday listening situations. This paper explores the role of cognition in hearing aid use and describes recent work aimed at determining individual cognitive spare capacity, or the ability to process speech heard in noise in ways that may be relevant for communication.

Key words

Cognition, working memory, cognitive spare capacity, hearing aids, signal processing Abbreviations

NR Binary masking Noise Reduction

ELU Ease of Language Understanding

WDRC Wide Dynamic Range Compression 


\section{Cognitive Spare Capacity}

The importance of cognitive skills for the successful use of advanced signal processing algorithms in modern digital hearing aids was identified a decade ago ${ }^{1}$. In 2003 , two papers from different research groups demonstrated a relationship between cognition and successful listening with hearing aids ${ }^{2,3}$. In those studies, it was amplitude compression that was at the focus of attention. Later studies have extended this finding to other kinds of signal processing, indicating that good cognitive skills may be a prerequisite for benefitting from any kind of advanced hearing aid signal processing ${ }^{4,5}$.

The earliest studies and several that followed them ${ }^{6-12}$ investigated the role of cognition in hearing aid benefit by studying how hearing aid outcomes, such as speech perception in noise performance and subjectively rated effort, correlated with independent measures of memory and executive function, often administered in the visual modality. Visual measures of cognition have the advantage of bypassing the impaired auditory modality. Thus, they tap into a pool of resources that are believed to be largely modality independent ${ }^{13}$. However, in a seminal paper in 2006, Kathy Pichora-Fuller pointed out the importance of studying the individual's ability to deploy cognitive resources to store and process what they had actually heard. Because we use our cognitive resources in the very act of listening, especially in noise, the cognitive capacity that remains to process heard material is lower than the cognitive capacity measured by independent visual tasks. This residual cognitive resource, we call cognitive spare capacity ${ }^{14}$. In the present paper, we will discuss why cognition is an important factor in gaining benefit from advanced signal processing algorithms in modern digital hearing aids and why it is important to measure cognitive spare capacity. We will also describe tests of cognitive spare capacity that are being used in a research context and how versions of these may eventually be applied in the clinic.

Cognition refers to thinking and memory. Thinking includes active processing of information from the senses including both what we hear and see as well as what we smell, taste and 


\section{Cognitive Spare Capacity}

touch. We can also think about what we have experienced in the past and plan for the future. Information about past experiences including when and where they happened is stored in episodic long-term memory along with our personal plans for the future while general world knowledge including the lexical items and grammatical structure of our language is stored in semantic long-term memory ${ }^{15}$. Thus, thinking and memory are inextricably linked. Thinking takes place in working memory. Working memory is the capacity to store information for a short period and process it on line ${ }^{14,16}$; a good example is encoding the words of a target speaker and assembling them to make sense while at the same time preparing an appropriate answer. Before words can be encoded into memory they need to be decoded from the input signal. Decoding involves matching the incoming signal to representations stored in the lexicon in semantic long-term memory. Under ideal conditions this takes place rapidly and automatically but in adverse conditions more effortful processing needs to be invoked. Adverse conditions may include distortion of the signal at source such as a speaker with a foreign accent or a speech impediment or when received as a result of hearing impairment or hearing aid signal processing ${ }^{17}$. Decoding the signal under adverse conditions may require storing unidentified fragments of the speech signal in working memory until they can be disambiguated $^{13}$. At the same time as unidentified fragments of the speech signal have to be stored, processing has to continue. For example, the contents of working memory need to be continually updated with the latest information while older items that may or may not have been successfully decoded need to be discarded ${ }^{18}$. The smooth function of working memory is also facilitated by inhibiting irrelevant information from entering working memory ${ }^{19}$. The extra working memory storage and processing load generated by trying to understand speech in noise pushes the limits of available capacity and is experienced as effortful ${ }^{11}$.

Working memory is characterised by its individual variation ${ }^{14,20}$, see Figure 1 . This variation is apparent between individuals but it is also evident within one and the same individual in 


\section{Cognitive Spare Capacity}

different situations and at different times of life $\mathrm{e}^{20}$. The capacity of working memory develops during childhood ${ }^{21}$ and declines in old age ${ }^{22}$. Throughout the life span, individual differences in working memory capacity are associated with the ability to perform language-related tasks that require effortful processing. In childhood, working memory is associated with developing reading ability ${ }^{23,24}$. This association falls off once reading ability is established ${ }^{23}$. However, the ability to understand speech in adverse conditions is generally associated with working memory capacity ${ }^{25}$. Working memory can be conceptualized as a general cognitive resource that operates at the focus of attention ${ }^{26}$. However, modular approaches have also been proposed $^{16,27}$. General resource models focus on the trade-off between storage and processing capacity; the general idea being that if you store a lot of information in working memory there is less capacity available for processing and vice versa if the task in hand needs a lot of processing.

Figure 1 about here

The classic modular approach to working memory goes back to Baddeley and Hitch in $1974^{27}$. Early versions of this model included a central executive that deployed cognitive resources to two slave buffers: the phonological loop and the visuospatial sketchpad and more recently an episodic buffer was added ${ }^{16}$. In the context of language and communication, the phonological loop has received much attention, but there is growing interest among the scientific community for the episodic buffer whose function is the integration and storage of multimodal information from different sources. An episodic buffer for the Rapid Automatic Multimodal Binding of PHOnology (RAMBPHO) is at the heart of the working memory model for Ease of Language Understanding (ELU) ${ }^{13,28,29}$. This model has proved to be particularly fruitful in the field of Cognitive Hearing Science ${ }^{30}$. In particular, it predicts that in adverse listening conditions, explicit or conscious processing resources will be brought into play to achieve language understanding and that this may be perceived as effortful. There is 


\section{Cognitive Spare Capacity}

now a substantial amount of support for the model, not least relating to the ability of persons with hearing impairment to benefit from advanced signal processing in digit hearing aids (Rönnberg et al., 2013).

The first evidence of the relationship between cognitive skills and listening with advanced signal processing ${ }^{3,7-9}$ related to Wide Dynamic Range Compression (WDRC) which adjusts amplification to the intensity of the acoustic signal so that soft sounds become audible without loud sounds becoming too loud $\mathrm{d}^{31}$. This kind of signal processing is available in most modern digital hearing aids. Fast-acting compression release (less than 200 milliseconds) distorts the speech signal by altering the temporal envelope ${ }^{32}$ but allows the listener to hear target information in the weaker dips in a modulated noise masker. Slow-acting compression release (longer than 200 milliseconds), on the other hand, does not distort the signal to the same extent, but may under-amplify soft sounds that immediately follow loud ones. The pros and cons of WDRC have been reviewed by Moore ${ }^{33}$.

Lunner $^{2}$ found that persons with higher cognitive capacity were better able than persons with lower cognitive capacity to identity the effects of WDRC on the noisy speech signal delivered by an experimental hearing aid, while Gatehouse et al. ${ }^{3,8}$ showed that the benefit of WDRC in terms of the ability to perceive speech in modulated noise was dependent on cognitive capacity. Lunner and Sundewall-Thorén ${ }^{9}$ extended the findings of Gatehouse et al ${ }^{3,8}$ by showing that while hearing aids users with high cognitive capacity benefited from fast-acting compression, those with lower cognitive capacity actually performed better with slow-acting compression. Indeed, Lunner and Sundewall-Thorén ${ }^{9}$ demonstrated that although hearing thresholds may explain a substantial portion of individual variation in the ability to understand speech in steady state noise with slow-acting compression, with little contribution of cognitive ability, the opposite is true in modulated noise with fast-acting compression. In the challenging conditions constituted by listening to speech with fast-acting compression 


\section{Cognitive Spare Capacity}

against a background of modulated noise, cognitive capacity explained almost $40 \%$ of the variance in performance while hearing thresholds explained less than $5 \%$ of the remaining variance, see Figure 2. This pattern was interpreted as indicating that when the demands of a listening situation are relatively low, even moderate cognitive storage and processing capacity is sufficient to process audible information. However, when listening demands are high, persons with good working memory capacity have an advantage. High listening demands may include having to deal with background talkers and distortion of the speech signal by aggressive signal processing. Under such conditions, the full storage and processing capacity of working memory may have to be brought into play to achieve speech comprehension ${ }^{5,28}$.

Figure 2 about here.

This line of argument is in agreement with the ELU model ${ }^{13,28,29}$. The ELU model explains this phenomenon in terms of Rapid Automatic Multimodal Binding of PHOnology (RAMBPHO) in the episodic buffer of working memory. When the incoming speech signal can be matched rapidly and automatically to existing phonological representations in the lexicon, speech understanding takes place with relative ease. However, when listening conditions are suboptimal, for example when the signal is degraded or distorted or when phonological representations in semantic long term memory are degraded due to severe longterm hearing impairment ${ }^{34}$, explicit cognitive processing resources have to be brought into play to cope with mismatch. In particular, when hearing aid signal processing distorts the signal in an interaction with modulated background noise, cognitive resources are probably required to integrate fragments of the target signal glimpsed in the dips in modulated noise and match them to existing representations ${ }^{13}$.

Different measures have been used to tap into cognitive capacity in different studies. The Gatehouse et al. studies ${ }^{3,8}$ as well as the study by Lunner and Sundewall-Thorén ${ }^{9}$ used a 


\section{Cognitive Spare Capacity}

cognitive measure based on monitoring tasks that required the identification of specific threecharacter sequences embedded in longer sequences ${ }^{35}$. In his 2003 paper $^{2}$, Lunner employed two cognitive measures: the reading span task and a phonological processing task, and found that the reading span task was the stronger predictor of both aided and unaided performance on a test of speech perception in noise after age and hearing thresholds were controlled for.

Reading span and a letter monitoring task were used by Foo et al. ${ }^{7}$ in a study investigating the ability to recognize speech in noise with new WDRC settings. The results showed that speech recognition in noise performance using both the Hagerman sentences ${ }^{36,37}$ and the Swedish Hearing In Noise Test (HINT) ${ }^{38}$ correlated consistently with performance on the reading span task, irrespective of whether noise was modulated or not and irrespective of whether the new hearing aid settings included fast or slow time constants. Furthermore, the Hagerman sentence correlations with reading span remained robust when hearing threshold was corrected for. Performance on the letter monitoring task, on the other hand, was not such a reliable predictor of speech recognition performance, even though there was a moderately strong significant correlation $(\mathrm{r}=.38)$ between performance on the two cognitive tasks. Both the reading span task and the character monitoring tasks are characterized by their storage and processing demands: information has to be kept in mind and processed, which is a defining feature of working memory ${ }^{14,16}$. However, Foo et al. ${ }^{7}$ argued that whereas the reading span task requires simultaneous storage and processing these demands are more serial in a monitoring task. A further aspect in that particular study was that the target three-character sequences were unambiguously lexical items in the Swedish language with controlled frequency, making lexical decision relatively easy. In previous studies, these parameters may have not been as well controlled, making monitoring tasks harder and more cognitively taxing and thus more predictive of success on other cognitively demanding tasks. A growing body of literature 


\section{Cognitive Spare Capacity}

shows that the reading span task is a potent predictor of successful listening in adverse conditions $^{25}$.

The reading span task was originally developed by Daneman and Carpenter ${ }^{14}$. In this first version of the task, participants had to read series of sentences aloud and then recall the final word of each sentence at the end of a set. The sentences were between 13 and 16 words long and were semantically plausible. The first set of sentences consisted of two sentences only. This was followed by two further sets of two sentences each. The next three sets consisted of three sentences. Set size increased by one sentence at a time up to a maximum of six sentences. Performance on this task was found to correlate with a range of reading comprehension measures in college students, whereas digit and word span measures did not predict comprehension. A refinement of this concept was introduced by Baddeley, Logie, Nimmo-Smith and Brereton in their 1985 study $^{39}$. Here participants listened to sentences, so it was not a reading-based test, however, certain critical aspects of the task were subject to new experimental control or manipulation. The sentences were short, including, for example, person, verb and object, and only half of them were semantically plausible. Participants had to categorise each sentence as it was presented on the basis of whether it made sense or not and at the end of each set of sentences they were cued to report either the person or the object. A text-based version of the concept developed by Baddeley et al. ${ }^{39}$ was used in a study by Rönnberg, Lyxell, Arlinger \& Kinnefors ${ }^{40}$ that studied the relationship between speechreading and cognitive function in hearing impaired individuals. In the reading span test, the participants read sentences that were presented one word at a time on a computer screen. First, they had to determine whether the sentence made sense and then they had to report the final words of each sentence in a particular set. In the Rönnberg et al. study ${ }^{40}$, reading span performance was found to be associated with the latency of the P200 component of visually evoked potentials. Coincidentally, enhanced auditory P200's have also been tied to cognitive 


\section{Cognitive Spare Capacity}

processes involved in auditory learning (Tremblay et al. 2009; Ross and Tremblay 2009).

This version of the task and similar implementations have been used in many of the studies from our lab investigating the role of cognition in listening in noise with and without hearing aids $^{7,9-12,41,42}$. In recent studies, shortened versions of the task including sentence set sizes ranging from three to five ${ }^{43}$ or $\operatorname{six}^{44}$ and with only two sets per size have been used. All versions of the task described here share simultaneous storage and processing demands. Sentences have to be kept in mind while their semantic content is processed.

A growing literature shows the predictive value of the reading span task in relation to the ability to benefit from a range of different hearing aid signal processing algorithms. While Foo et al. ${ }^{7}$ and Rudner et al. ${ }^{10}$ showed that reading span performance was associated aided speech recognition performance in noise with new compression settings, irrespective of whether compression was fast- or slow-acting, Rudner et al. ${ }^{41}$ extended these findings across languages from Swedish to Danish, demonstrating that they were not language specific. These findings are in line with the prediction of the ELU model that in the adverse listening conditions that arise for a person with hearing impairment listening in noise, especially with new hearing aids settings, that understanding speech in noise will be associated with the engagement of the explicit cognitive processing capacity tapped by the reading span task. Rudner, Rönnberg and Lunner ${ }^{12}$ showed that even when individuals with hearing impairment have had the opportunity to become accustomed to WDRC, there is still an association with reading span in modulated noise at low signal to noise ratios with fast-acting compression. They also showed that this association is specific to the nature of the target speech material. In particular, the association was found for the stereotyped Swedish Hagerman sentences ${ }^{36}$ (e.g. "Lisa had eight red bowls") but not for the more natural type of sentence material in the Swedish Hearing In Noise Test ${ }^{38}$ (HINT; e.g. "The lady went shopping once a week"). This was in contrast to the findings of Foo et al. ${ }^{7}$, showing that reading span predicted speech 


\section{Cognitive Spare Capacity}

recognition in noise performance with both the Hagerman sentences and the HINT material when WDRC fittings were new. Having heard the first part of a sentence, it is easier to predict how it will end if the sentence is semantically coherent. Thus, it is harder to make predictions about the ending of Hagerman sentences than of HINT sentences. The results of Rudner et al. ${ }^{12}$ suggest that even when hearing aids wearers have become accustomed to the distortion of the speech signal caused by WDRC, they still need to engage explicit cognitive process to make sense of speech in noise if there is no help to be had from sentence coherence.

Other work shows that individual working memory capacity is important for making use of context. Zekveld et al. ${ }^{45}$ showed that reading span performance is associated with the ability to make good use of semantically related text cues when listening to speech in noise. Further Zekveld et al. (in prep) showed that the benefit obtained from text cues while listening to target speech masked by interfering speech is predicted by reading span performance. This applied both to hearing the words and remembering them. Thus, cognitive skills are not only related to the ability to perceive speech but also to the ability to use context to generate predictions to facilitate speech perception and the ability to encode heard speech into longterm memory.

It is clear beyond any shade of doubt that if we are to understand how hearing aid signal processing algorithms can benefit individual hearing aids wearers we need to take account of their cognitive skills. However, simply measuring working memory capacity may not be enough. Speech communication is a complex process that requires perception of the speech signal and processing of that signal to achieve language understanding. As we have seen, language understanding may involve integration of information from many sources. This takes place in the episodic buffer of working memory ${ }^{13,16,28,29}$. Hearing aids are designed for better audibility but also listening comfort and reduced listening effort ${ }^{4,5}$. In order to find out whether specific signal processing algorithms not only improve audibility but also facilitate 


\section{Cognitive Spare Capacity}

the processes involved in language understanding resulting in more comfort and less effort we need to be able to tap into the cognitive capacity that remains once successful hearing has taken place ${ }^{44}$. This is the concept of cognitive spare capacity ${ }^{46}$.

In a seminal study, Sarampalis et al. ${ }^{47}$ showed that the kind of signal processing used in hearing aids for reducing background noise could release cognitive resources for better memory encoding of speech heard in noise. However, at the time this was shown only for listeners with normal hearing We have recently shown that binary masking noise reduction $(\mathrm{NR})^{48}$ can provide a similar benefit for adults with hearing impairment ${ }^{43}$. Hearing impaired listeners who were experienced hearing aid users listened to sets of eight Swedish HINT sentences $^{38}$ either in quiet or at a signal to noise ratio that was individually adjusted to provide about $95 \%$ intelligibility. After each sentence they repeated the final word and at the end of each set of sentences they were prompted to recall the final words of all the eight sentences in any order. Importantly, we found that although background noise reduced recall performance, NR counteracted this effect. Further, the ability to benefit from NR in terms of better recall of speech in noise was related to reading span performance, specifically when the noise background was modulated, consisting of four competing talkers. Good working memory capacity measured by the reading span task ${ }^{14,39,40}$ boosted the ability to recall the words that had been heard most recently and were still retained in working memory rather than words that could only be successfully recalled because they had already been transferred to longterm memory.

In a second study ( $\mathrm{Ng}$ et al., in prep.), we focused on the memory facilitation for persons with hearing impairment provided by NR when target speech was presented against a multi-talker background and investigated whether this facilitation was influenced by whether the semantic content of the background noise was accessible or not; the competing talkers either spoke Swedish, just like the target talkers or Cantonese, a language unfamiliar to the participants in 


\section{Cognitive Spare Capacity}

the study. We also reduced the overall memory load by decreasing the number of sentences that had to be held in mind before recall took place from eight to seven. Again, we found that NR facilitated recall performance and that this effect was most prominent for sentences heard most recently and thus still retained in working memory.

We also found that Cantonese competing talkers interfered less with recall of target words than Swedish competing talkers and, interestingly, that when NR was applied, the decrement in memory performance caused by the Swedish competing talkers was reduced to the level caused by their Cantonese counterparts. This set of findings demonstrates that even when speech is fully audible, advanced signal processing can counteract the negative effects of background noise on the ability of persons with hearing impairment to remember what has been said. As we have found in previous studies, the effects of signal processing seem to be mediated by working memory capacity and the differential effects of NR on comprehensible and incomprehensible background babble suggest that it supports inhibition of irrelevant information.

In these NR studies ${ }^{43}$ (Ng et al., in prep.) we made a first step towards a test of Cognitive Spare Capacity by measuring memory performance for heard material and we found that memory for heard material can be improved by NR even when audibility is ensured. To take a more analytical approach to the concept of cognitive spare capacity, we initiated another set of studies in which we developed a new tool for measuring the storage and processing capabilities, not of working memory per se but of that portion of cognitive resource that remains once successful listening has taken place. This tool is known as the Cognitive Spare Capacity Test or $\mathrm{CSCT}^{44}$. At present, it is only available for research purposes, but we see good opportunities for adapting it for use as a clinical instrument in the future. The CSCT measures the ability to store different amounts of heard information and process it appropriately. These are the very skills that are required for successfully holding a meaningful 


\section{Cognitive Spare Capacity}

conversation. Once we have perceived the words our communicative partner is uttering, we need to keep them in mind while we ponder on their meaning and integrate them with our prior knowledge and intentions before delivering a response. This is quite a different matter from a speech perception in noise test that requires repeating back a sentence that we have more or less heard.

The CSCT provides the opportunity to measure not only memory storage of heard material but also different kinds of cognitive processing and how these are influenced by factors such different types of noise and different hearing aid signal processing algorithms. In the $\mathrm{CSCT}^{44}$, participants listen to lists of 13 two-digit numbers some of which are spoken by a man and some by a woman. The participants are instructed to memorise two particular numbers according to instructions. These tasks are designed to induce different kinds of executive processing: updating and inhibition. In one version of the updating task, the participant has to listen for and remember the highest number in the list spoken by the man and the highest number in the list spoken by the woman. Because the numbers are presented in serial order but not numerical order, the participant has to update items stored from the beginning of the list when higher numbers are encountered later in the list. At the end of the list the participants has to recall the appropriate numbers. In one version of the inhibition task, the participant has to listen for and remember odd numbers, but only when they are spoken by the man. This means that odd numbers spoken by the woman have to be inhibited. Sometimes the participant is instructed to additionally remember the first item in the list. This additional item can be added to both updating and inhibition tasks and induces an extra memory load that potentially interferes with executive processing.

Results to date ${ }^{44}$ (Mishra et al., in prep) show that performance is consistently better in low memory load conditions (without the additional item) than in high memory load conditions (with the additional item) and in inhibition task compared to the updating task. This applies to 


\section{Cognitive Spare Capacity}

persons with and without hearing impairment. We have also been able to show that performance on the updating and inhibition tasks of the CSCT correlate with independent visually based measures of updating and inhibition. Together, these results provide an important cognitive baseline in terms of memory storage and processing. We also find that performance is better in quiet than in noise for individuals with and without hearing impairment (Mishra et al., in prep.). In noisy conditions, CSCT performance is better when participants can see the speaker's face. Again, this applies to individuals both with and without hearing impairment. However, when the CSCT is performed in quiet we find the rather surprising effect that performance is better when the participants with normal hearing are not looking at the speaker's face, probably because the visual information provides a distraction rather than helping to provide better memory representations ${ }^{44}$ (Mishra et al., in prep.). This effect was not, however, found for participants with hearing impairment who showed no difference in performance in quiet with and without faces (Mishra et al., in prep.). This finding suggests that although watching the speaker's face can be a disadvantage for individuals with normal hearing during a cognitive task based on auditory stimuli, this is not the case of persons with hearing impairment. Interestingly, CSCT performance does not correlate consistently with working memory capacity measured using the reading span task. Indeed, both for participants with normal hearing and participants with hearing impairment we have found no consistent association between CSCT and reading span performance ${ }^{44}$ (Mishra et al., in prep). This suggests that CSCT is not just another way of measuring general working memory capacity but does indeed tap into a qualitatively and quantitatively different cognitive resource ${ }^{46}$.

The next step is to use the CSCT to evaluate the effects of advanced hearing aid signal processing on the ability to store and process heard information. It has been convincingly shown that working memory capacity is associated with the ability to benefit from advanced 


\section{Cognitive Spare Capacity}

hearing aid signal processing. By measuring cognitive spare capacity for speech heard in noise with different hearing aid settings using the CSCT, we will be able to determine whether the downstream effects of signal processing impact storage or processing or both. Indeed we will be able to separate effects of signal processing in high and low load conditions as well as when processing involves updating and inhibition. Thus, we will be able to show not just how signal processing influences speech perception in noise but the way in which it interacts with the storage and cognitive processing of speech-based representations. This will provide us with a window on how hearing aid signal processing plays into the real-life cognitive demands of communication.

We also envisage the possibility of developing a simplified version of CSCT for use in the clinic in connection with the fitting and evaluation of hearing aids. A simple version of CSCT would allow the audiologist to compare the effects of different signal processing regimes for the individual listener in terms of how they facilitate or interfere with cognitive abilities that are fundamental to good communication. We believe that the simplified CSCT will provide a valuable complement to standard audiological tests as well other tools becoming available to the audiologist including speech perception in noise testing and the reading span task. While the reading span task can measure cognitive abilities that are important for speech understanding in noise, the CSCT measures the ability to store and process heard information. This can help us better understand how individuals function in communicative situations with and without their hearing aids. 
Cognitive Spare Capacity

\section{References}

1. Pichora-Fuller MK. How cognition might influence hearing aid design, fitting, and outcomes. Hearing Journal. 2009;62(11):32-35.

2. Lunner T. Cognitive function in relation to hearing aid use. International Journal of Audiology. 2003;42(SUPPL. 1):S49-S58.

3. Gatehouse S, Naylor G, Elberling C. Benefits from hearing aids in relation to the interaction between the user and the environment. International Journal of Audiology. 2003;42(SUPPL. 1):S77S85.

4. Edwards B. The future of hearing aid technology. Trends in Amplification. 2007;11(1):31-45.

5. Lunner T, Rudner M, Rönnberg J. Cognition and hearing aids. Scand J Psychol. 2009;50(5):395-403.

6. Cox RM, Xu J. Short and long compression release times: Speech understanding, real-world preferences, and association with cognitive ability. J Am Acad Audiol. 2010;21(2):121-138.

7. Foo C, Rudner M, Rönnberg J, Lunner T. Recognition of speech in noise with new hearing instrument compression release settings requeres explicit cognitive storage and processing capacity. J Am Acad Audiol. 2007;18(7):618-631.

8. Gatehouse S, Naylor G, Elberling C. Linear and nonlinear hearing aid fittings - 2. patterns of candidature. International Journal of Audiology. 2006;45(3):153-171.

9. Lunner T, Sundewall-Thorén E. Interactions between cognition, compression, and listening conditions: Effects on speech-in-noise performance in a two-channel hearing aid. J Am Acad Audiol. 2007;18(7):604-617. 


\section{Cognitive Spare Capacity}

10. Rudner M, Foo C, RÖnnberg J, Lunner T. Cognition and aided speech recognition in noise: Specific role for cognitive factors following nine-week experience with adjusted compression settings in hearing aids. Scand J Psychol. 2009;50(5):405-418.

11. Rudner M, Lunner T, Behrens T, Thorén ES, Rönnberg J. Working memory capacity may influence perceived effort during aided speech recognition in noise. J Am Acad Audiol. 2012;23(8):577-589.

12. Rudner M, Rönnberg J, Lunner T. Working memory supports listening in noise for persons with hearing impairment. J Am Acad Audiol. 2011;22(3):156-167.

13. Rönnberg J, Rudner M, Foo C, Lunner T. Cognition counts: A working memory system for ease of language understanding (ELU). International Journal of Audiology. 2008;47(SUPPL. 2):S99-S105.

14. Daneman, Meredyth \& Carpenter, Patricia A. Individual differences in working memory and reading. Journal of Verbal Learning and Verbal Behavior. 1980;19:450-466.

15. Tulving E, ed. Episodic memory: From mind to brain. ; 2002Annual Review of Psychology; No. 53.

16. Baddeley A. The episodic buffer: A new component of working memory? Trends Cogn Sci (Regul $E d) .2000 ; 4(11): 417-423$.

17. Mattys SL, Davis MH, Bradlow AR, Scott SK. Speech recognition in adverse conditions: A review. Language and Cognitive Processes. 2012;27(7-8):953-978.

18. Rudner M, Rönnberg J, Lunner T. Working memory supports listening in noise for persons with hearing impairment. J Am Acad Audiol. 2011;22(3):156-167.

19. Sörqvist P. The role of working memory capacity in auditory distraction: A review. Noise and Health. 2010;12(49):217-224. 


\section{Cognitive Spare Capacity}

20. Pichora-Fuller MK. Audition and cognition: What audiologists need to know about listening. In:

Palmer, C. \& Seewald, R., ed. Hearing care for adults. Phonak: Stäfa, Switzerland; 2007:71-85.

21. Engel de Abreu PMJ, Conway ARA, Gathercole SE. Working memory and fluid intelligence in young children. Intelligence. 2010;38(6):552-561.

22. Nyberg L, Lövdén $M$, Riklund $K$, Lindenberger $U$, Bäckman L. Memory aging and brain maintenance. Trends Cogn Sci (Regul Ed ). 2012;16(5):292-305.

23. Gathercole SE, Pickering SJ, Knight C, Stegmann Z. Working memory skills and educational attainment: Evidence from national curriculum assessments at 7 and 14 years of age. Applied Cognitive Psychology. 2004;18(1):1-16.

24. Baddeley A, Gathercole S, Papagno C. The phonological loop as a language learning device. Psychol Rev. 1998;105(1):158-173.

25. Akeroyd MA. Are individual differences in speech reception related to individual differences in cognitive ability? A survey of twenty experimental studies with normal and hearing-impaired adults. International Journal of Audiology. 2008;47(SUPPL. 2):S53-S71.

26. Cowan N. The focus of attention as observed in visual working memory tasks: Making sense of competing claims. Neuropsychologia. 2011;49(6):1401-1406.

27. Baddeley AD, Hitch G, eds. Working memory. ; 1974Psychology of Learning and Motivation Advances in Research and Theory; No. 8.

28. Rönnberg J, Rudner M, Lunner T, Zekveld AA. When cognition kicks in: Working memory and speech understanding in noise. Noise and Health. 2010;12(49):263-269. 


\section{Cognitive Spare Capacity}

29. Rönnberg J. Cognition in the hearing impaired and deaf as a bridge between signal and dialogue: A framework and a model. International Journal of Audiology. 2003;42(SUPPL. 1):S68-S76.

30. Arlinger S, Lunner T, Lyxell B, Kathleen Pichora-Fuller M. The emergence of cognitive hearing science. Scand J Psychol. 2009;50(5):371-384.

31. Souza PE. Effects of compression on speech acoustics, intelligibility, and sound quality. Trends in Amplification. 2002;6(4):131-165.

32. Jenstad LM, Souza PE. Temporal envelope changes of compression and speech rate: Combined effects on recognition for older adults. Journal of Speech, Language, and Hearing Research. 2007;50(5):1123-1138.

33. Moore BCJ. The choice of compression speed in hearing aids: Theoretical and practical considerations and the role of individual differences. Trends in Amplification. 2008;12(2):103-112.

34. Classon E, Rudner M, Rönnberg J. Working memory compensates for hearing related phonological processing deficit. J Commun Disord. 2013;46(1):17-29.

35. Knutson JF, Schartz HA, Gantz BJ, Tyler RS, Hinrichs JV, Woodworth G. Psychological change following 18 months of cochlear implant use. Annals of Otology, Rhinology and Laryngology. $1991 ; 100(11): 877-882$

36. Hagerman B, Kinnefors C. Efficient adaptive methods for measuring speech reception threshold in quiet and in noise. Scand Audiol. 1995;24(1):71-77.

37. Hagerman B. Sentences for testing speech intelligibility in noise. Scand Audiol. 1982;11(2):79-87.

38. Hällgren M, Larsby B, Arlinger S. A swedish version of the hearing in noise test (HINT) for measurement of speech recognition. International Journal of Audiology. 2006;45(4):227-237. 


\section{Cognitive Spare Capacity}

39. Baddeley A, Logie R, Nimmo-Smith I, Brereton N. Components of fluent reading. Journal of Memory and Language. 1985;24(1):119-131.

40. Ronnberg J, Arlinger S, Lyxell B, Kinnefors C. Visual evoked potentials: Relation to adult speechreading and cognitive function. J Speech Hear Res. 1989;32(4):725-735.

41. Rudner M, Foo C, Sundewall-Thorén E, Lunner T, Rönnberg J. Phonological mismatch and explicit cognitive processing in a sample of 102 hearing-aid users. International Journal of Audiology. 2008;47(SUPPL. 2):S91-S98.

42. Zekveld AA, Rudner M, Johnsrude IS, Heslenfeld DJ, Rönnberg J. Behavioral and fMRI evidence that cognitive ability modulates the effect of semantic context on speech intelligibility. Brain Lang. 2012;122(2):103-113.

43. Ng EHN, Rudner M, Lunner T, Syskind Pedersen M, Rönnberg J. <br />Improved cognitive processing of speech for hearing aid users with noise reduction. International Journal of Audiology. 2013.

44. Mishra S, Lunner T, Stenfelt S, Rönnberg, J. \& Rudner, M. Visual information can hinder working memory processing of speech. Journal of Speech, Language, and Hearing Research. 2013.

45. Zekveld AA, Rudner M, Johnsrude IS, Festen JM, Van Beek JHM, Rönnberg J. The influence of semantically related and unrelated text cues on the intelligibility of sentences in noise. Ear Hear. 2011;32(6):e16-e25.

46. Rudner M, Ng EHN, Rönnberg N, Mishra S, Rönnberg J, Lunner, T. \& Stenfelt, S. Cognitive spare capacity as a measure of listening effort. Journal of Hearing Science. 2011;1(2):47-49. 
Cognitive Spare Capacity

47. Sarampalis A, Kalluri S, Edwards B, Hafter E. Objective measures of listening effort: Effects of background noise and noise reduction. Journal of Speech, Language, and Hearing Research.

2009;52(5):1230-1240.

48. Wang D, Kjems U, Pedersen MS, Boldt JB, Lunner T. Speech intelligibility in background noise with ideal binary time-frequency masking. J Acoust Soc Am. 2009;125(4):2336-2347.

Auditory training alters the physiological detection of stimulus-specific cues in humans.

Tremblay KL, Shahin AJ, Picton T, Ross B.

Clin Neurophysiol. 2009 Jan;120(1):128-35.

Stimulus experience modifies auditory neuromagnetic responses in young and older listeners. Ross B, Tremblay K.

Hear Res. 2009 Feb;248(1-2):48-59

Jerker Rönnberg, Thomas Lunner, Adriana Zekveld, Patrik Sörqvist,Henrik Danielsson, Björn Lyxell, Örjan Dahlström, Carine Signoret, Stefan Stenfelt, M. Kathleen Pichora-Fuller \& Mary Rudner. (2013). The Ease of Language Understanding (ELU) model: Theoretical, empirical, and clinical advances. Frontiers in Systems Neuroscience (under revision) 


\section{Cognitive Spare Capacity}

\section{Figure captions}

Figure 1. Schematic depictions of inter-individual differences in working memory span showing that two people may differ in their total working memory capacity (a), and intra-individual differences showing that for any given individual the allocation of the person's limited capacity to the processing and storage functions of working memory varies with task demands such as when the level of background noise increases during listening (b) (reprinted from Pichora-Fuller, 2007 with permission).

Figure 2. Explained variance in speech recognition in noise for different test conditions of background noise and hearing aid time constants. Visual letter monitoring is the cognitive test and the pure-tone average of six frequencies is the measure of audiometric hearing loss (reprinted with permission from the American Academy of Audiology). 
Figure 1

a. Inter-individual differences in working memory capacity

Fred



Mary

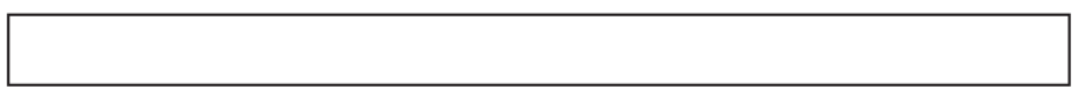

b. Intra-individual differences in working memory span (WMS): Allocation of resources to processing vs storage varies with tas
Fred in quiet

Processing Storage
Fred in noise

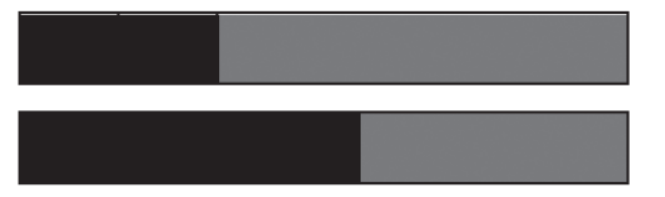
WMS $=6$
Fred in more noise
WMS $=4$
WMS $=2$ 
Figure 2
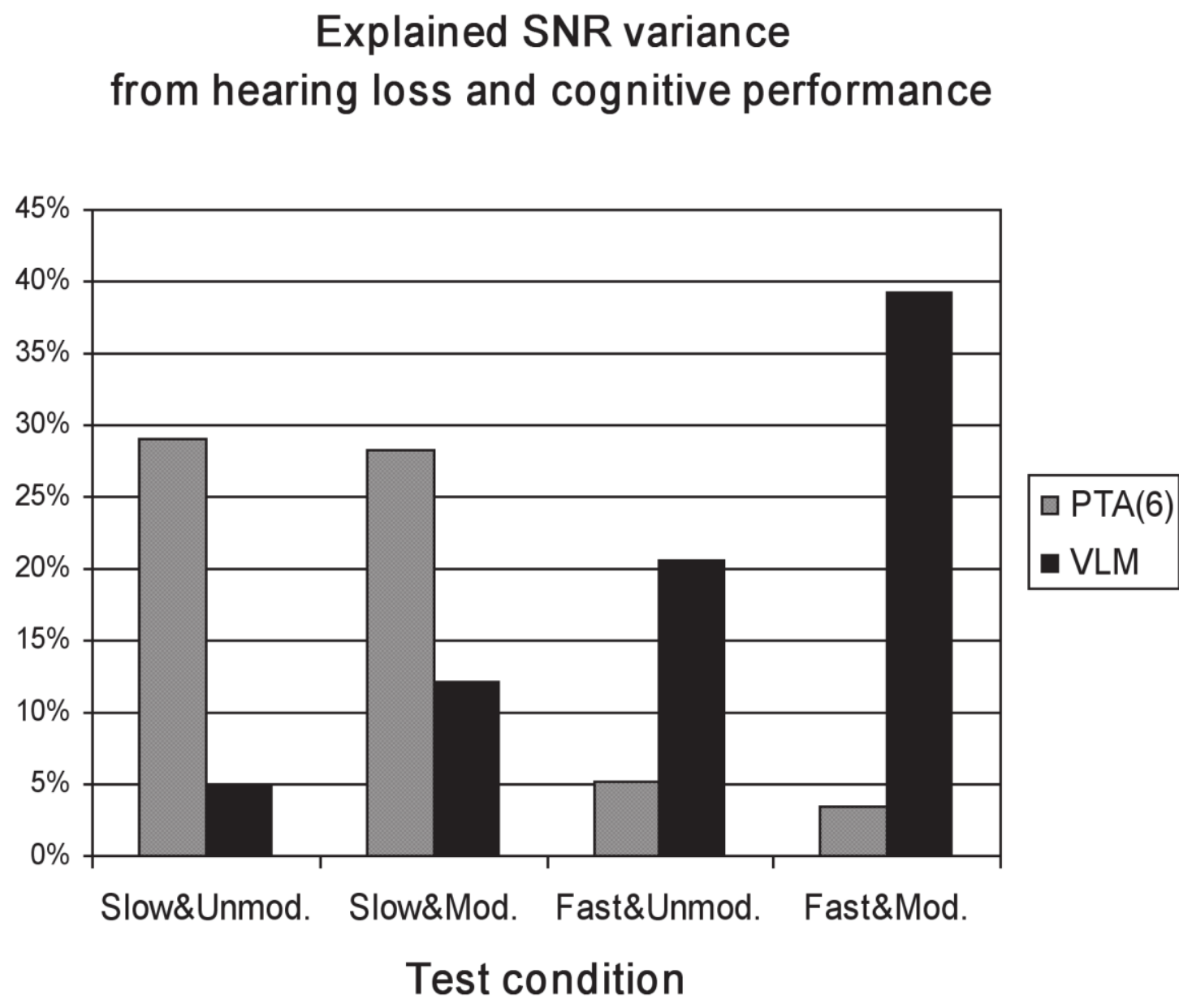
Cognitive Spare Capacity 